\title{
CCR10 expression is a common feature of circulating and mucosal epithelial tissue IgA Ab-secreting cells
}

\author{
Eric J. Kunkel, ${ }^{1,2}$ Chang H. Kim, ${ }^{1,2}$ Nicole H. Lazarus, ${ }^{1,2}$ Mark A. Vierra, ${ }^{3}$ Dulce Soler, ${ }^{4}$ \\ Edward P. Bowman, ${ }^{5}$ and Eugene C. Butcher ${ }^{1,2}$ \\ ${ }^{1}$ Laboratory of Immunology and Vascular Biology, Department of Pathology, Stanford University School of Medicine, \\ Stanford, California, USA \\ ${ }^{2}$ The Center for Molecular Biology and Medicine, Veterans Affairs Palo Alto Health Care System, Palo Alto, California, USA \\ ${ }^{3}$ Department of Surgery, Stanford University School of Medicine, Stanford, California, USA \\ ${ }^{4}$ Millennium Pharmaceuticals Inc., Cambridge, Massachusetts, USA \\ ${ }^{5}$ DNAX Research Inc., Palo Alto, California, USA
}

The dissemination of IgA-dependent immunity between mucosal sites has important implications for mucosal immunoprotection and vaccine development. Epithelial cells in diverse gastrointestinal and nonintestinal mucosal tissues express the chemokine MEC/CCL28. Here we demonstrate that CCR10, a receptor for MEC, is selectively expressed by IgA Ab-secreting cells (large $\mathrm{s} / \mathrm{cIgA}^{+} \mathrm{CD} 38^{\text {hi }} \mathrm{CD} 19^{\text {int } /-} \mathrm{CD} 20^{-}$), including circulating $\operatorname{IgA}^{+}$plasmablasts and almost all $\operatorname{IgA}{ }^{+}$plasma cells in the salivary gland, small intestine, large intestine, appendix, and tonsils. Few T cells in any mucosal tissue examined express CCR10. Moreover, tonsil IgA plasmablasts migrate to MEC, consistent with the selectivity of CCR10 expression. In contrast, CCR9, whose ligand TECK/CCL25 is predominantly restricted to the small intestine and thymus, is expressed by a fraction of IgA $\mathrm{Ab}$-secreting cells and almost all $\mathrm{T}$ cells in the small intestine, but by only a small percentage of plasma cells and plasmablasts in other sites. These results point to a unifying role for CCR10 and its mucosal epithelial ligand MEC in the migration of circulating IgA plasmablasts and, together with other tissue-specific homing mechanisms, provides a mechanistic basis for the specific dissemination of IgA Ab-secreting cells after local immunization.

J. Clin. Invest. 111:1001-1010 (2003). doi:10.1172/JCI200317244.

\section{Introduction}

Epithelial tissues are continuously exposed to a wide variety of pathogens and toxins. The mammalian immune system has developed intricate mechanisms to provide defense at epithelial surfaces and control potentially harmful responses to normal symbiotic flora. These mechanisms include tightly controlled transport across the epithelium, resident phagocytes and antigen-presenting cells, and numerous $\mathrm{T}$ and $\mathrm{B}$ lymphocytes providing both cell-mediated and humoral immunity. At mucosal epithelial surfaces in particular, the local production and transepithelial transport of polymeric IgA secreted by resident plasma cells plays an important role in pathogen and toxin neutralization (1-3). Interestingly, local mucosal immunization leads to antigen-specific IgA production

Received for publication October 25, 2002, and accepted in revised form January 14, 2003.

Address correspondence to: Eric J. Kunkel, BioSeek Inc. 863-C Mitten Road Burlingame, California 94010, USA. Phone: (650) 231-1251; Fax: (650) 552-0725;

E-mail: ekunkel@bioseekinc.com.

Conflict of interest: The authors have declared that no conflict of interest exists.

Nonstandard abbreviations used: phycoerythrin (PE); peripheral blood mononuclear leukocyte (PBML); germinal center (GC); plasma cells (PCs); surface IgA (sIgA); allophycocyanin (APC); antibody secreting cell (ASC). at distant mucosal sites in both humans $(4,5)$ and mice $(6,7)$, likely through the migration of IgA-secreting B cells (8). These findings suggest that a simple nasal or oral vaccination $(1,9-11)$ may be effective at inducing IgA-dependent pathogen protection at multiple mucosal sites, in addition to systemic cell-mediated immunity. The development of efficient mucosal vaccines capable of generating disseminated $\mathrm{Ab}$-mediated protection requires an understanding of the fundamental molecular mechanisms of IgA-secreting B cell trafficking between distant mucosal sites.

Epithelial cells themselves are emerging as important players in the homeostatic trafficking of lymphocyte subsets, including Ab-secreting cells, through their secretion of tissue-specific chemokines (12). For instance, epithelial cells in the small intestine secrete the chemokine $\operatorname{TECK}(13,14)$, a ligand for the chemokine receptor CCR9 expressed on virtually all small intestinal $\mathrm{T}$ cells and on a subset of circulating mucosal memory T cells, as well (14, 15). In mice, TECK is also a chemoattractant for splenic and small-intestinal IgA Ab-secreting cells (16). Because CCR9 $9^{+}$lymphocytes are rare in the colon and virtually absent in other epithelial tissues $(14,17)$, CCR9 and TECK may serve to segregate and compartmentalize the small intestinal immune response. An analogous situation is the expression of the related chemokine CTACK/CCL27 by keratinocytes, the epithelial cells of the skin (18). CCR10, the receptor for CTACK, is 
expressed on circulating $\mathrm{CLA}^{+}$skin-homing lymphocytes $(18,19)$, and together with CCR4 and TARC/CCL17 (20) (and skin-selective adhesion receptors), determines the selectivity of skin lymphocyte trafficking.

We and others have recently identified a related CCR10 ligand, the chemokine MEC, that is expressed by epithelial cells in a variety of tissues, including the salivary gland, mammary gland, small and large intestines, and trachea $(21,22)$. MEC is also chemotactic for circulating CLA $^{+}$lymphocytes by virtue of being a CCR10 ligand $(21,22)$, but CLA ${ }^{+}$lymphocytes are virtually never found in gastrointestinal tissues because they do not express the $\alpha_{4} \beta_{7}$ integrin required for trafficking into these organs (via interaction with vascular MAdCAM-1 in intestinal sites) (23). The expression of CCR10 at the mRNA level in many of the same sites where MEC is expressed, including the small and large intestines (24), suggests the presence of an unidentified lymphocyte subset expressing CCR10.

Here, we show that CCR10 is expressed on IgA Absecreting cells, but not memory $\mathrm{T}$ or $\mathrm{B}$ cells, in the human gastrointestinal tract (i.e., stomach, small intestine, and colon), salivary gland, and mucosa-associated lymphoid tissues (i.e., tonsils and appendix). Tonsil plasmablasts expressing CCR10 chemotax to MEC and approximately $70 \%$ of circulating IgA plasmablasts express CCR10, suggesting that blood IgA plasmablasts can home to and localize within MECexpressing mucosal epithelial sites using CCR10. We also find that in the human, as in the murine system, CCR9 is expressed on a subset of circulating IgA plasmablasts and on a subset of IgA plasma cells within the small intestine, suggesting a more restricted role for CCR9 and TECK in small intestinal IgA immunity. Taken together, these results suggest a role for CCR10 and MEC in unifying the localization of IgA plasma cells to the physically dispersed organs of the secretory IgA immune system. Thus, it is likely that induction of CCR10 on pathogen-specific IgA-secreting B cells may be an important factor for the successful dissemination of IgA-dependent protection following local mucosal infection or vaccination.

\section{Methods}

$A b$ 's and reagents. Anti-human CCR $10 \mathrm{mAbs} 37$ (mouse $\mathrm{IgG}_{1}$ ), 1908 (mouse $\mathrm{IgG}_{1}$ ), and 1363 (mouse $\mathrm{IgG}_{2 \mathrm{a}}$ ) were made by immunizing mice with murine $\mathrm{BAF} / 3$ pro- $\mathrm{B}$ cells transfected with human CCR10, as described (19). Anti-human CCR10 mAb 1B5 (mouse $\operatorname{IgG}_{2 \mathrm{a}}$ ) was made by immunizing mice with human CCR10 transfected L1/ 2 cells (a pre-B cell line) at Millenium Pharmaceuticals Inc. (Cambridge, Massachusetts, USA). All four CCR10 mAb's exhibited virtually identical staining patterns on both blood and tissue lymphocytes when tested by flow-cytometry or immunohistochemistry. Antihuman CCR9 mAb's 3C3 (mouse $\operatorname{IgG}_{2 \mathrm{~b}}$ ) and 96-1 (mouse $\operatorname{IgG}_{1}$ ) have been described $(14,15)$. Unconjugated isotype controls were obtained from SigmaAldrich (St. Louis, Missouri, USA), and an IgG Igiotin $^{-}$ isotype control was obtained from PharMingen (San Diego, California, USA). Anti-human CCR6 mAb (mouse $\operatorname{IgG} \mathrm{G}_{2 \mathrm{~b}}$, clone 53103.111) was obtained from R\&D Systems Inc. (Minneapolis, Minnesota, USA). Directly conjugated mouse anti-human CD3-APC ( $\mathrm{IgG}_{1}$, clone UCHT1), CD4-phycoerythrin (CD4-PE) ( $\mathrm{IgG}_{1}$, clone RPA-T4), CD8-PE (IgG 1 , clone RPA-T8), CD45RA-FITC ( $\operatorname{IgG}_{2 \mathrm{~b}}$, clone HI100), CD20-APC ( $\operatorname{IgG}_{2 \mathrm{~b}}$, clone 2H7), CD19-PE (IgG 1 , clone HIB19), IgD-FITC ( $\operatorname{IgG}_{2 \mathrm{a}}$, clone $\left.1 \mathrm{A6}-2\right)$, CD14-FITC ( $\operatorname{IgG}_{2 \mathrm{a}}$, clone M5E2), CD94-FITC ( $\operatorname{IgG}_{1}$, clone HP-3D9), and CD38-FITC $\left(\operatorname{IgG}_{1}\right.$, clone HIT2) were from PharMingen. Antihuman $\operatorname{IgA}_{1 / 2}$-biotin $\left(\operatorname{IgG}_{1}\right.$, clone G20-359) and IgGbiotin ( $\operatorname{IgG}_{1}$, clone G18-145) were from PharMingen. EDTA was from Sigma-Aldrich.

Tissues and lymphocyte isolation. Normal human tonsil, stomach, jejunum, ileum, colon, and appendix were obtained from patients undergoing various surgical procedures. All human subject protocols were approved by the Institutional Review Board at Stanford University. Some tissues were obtained as frozen specimens in OCT from the Department of Pathology at Stanford University or the Cooperative Human Tissue Network (Western Division, Nashville, Tennessee, USA). Lymphocytes from the lamina propria of the human gastrointestinal tract were isolated as described previously (14) using no enzymatic treatment. Briefly, pieces of tissue were cut open, laid flat, and washed with ice-cold HBSS. The serosa was separated from the mucosa with scissors and discarded. The mucosa was cut into strips and then incubated in cold $1 \mathrm{mM}$ EDTA/HBSS with constant stirring for $30 \mathrm{~min}$ to remove the epithelium and intraepithelial lymphocytes (this step was repeated several times until no more epithelial shedding occurred). The remaining mucosal strips were crushed through a 50-mesh strainer (Sigma-Aldrich) to isolate lamina propria lymphocytes. Tonsil and appendix lymphocytes were isolated by dispersing the tissues through a wire mesh followed by hypotonic erythrocyte lysis. We have determined previously (14) that the nonenzymatic treatments used to isolate lymphocytes from these tissues do not affect chemokine receptor, adhesion molecule, or marker expression. Human peripheral blood mononuclear leukocytes (PBMLs) were isolated by Ficoll-gradient (Amersham Biosciences, Piscataway, New Jersey, USA) centrifugation. For analysis of circulating IgA plasmablasts, PBMLs were depleted of T cells before staining using anti-CD3 magnetic beads (Dynal Inc., Lake Success, New York, USA), according to the manufacturer's directions.

Immunohistochemistry. Frozen sections of various OCT-embedded tissues were fixed for $10 \mathrm{~min}$ in cold acetone, dried at room temperature for $1 \mathrm{~h}$, then rehydrated for $30 \mathrm{~min}$ in PBS. Tissues were then blocked with $25 \%$ goat serum for 10 min before incubation with primary (unconjugated CCR $10 \mathrm{mAb}$ 's, $10 \mu \mathrm{g} / \mathrm{ml}$ ) or secondary $\mathrm{Ab}$ (PE goat anti-mouse $\mathrm{F}\left(\mathrm{ab}^{\prime}\right)_{2}, 1: 100$; Jackson ImmunoResearch Laboratories Inc., West Grove, Pennsylvania, USA) for $40 \mathrm{~min}$ at room temperature. 
Biotinylated mouse anti-human Ig Ab's $(10 \mu \mathrm{g} / \mathrm{ml})$ were incubated for $40 \mathrm{~min}$ at room temperature and detected with a 1:150 dilution of SAv-FITC (Jackson ImmunoResearch Laboratories Inc.). Isotype control $A b$ 's were detected in an identical manner. Directly conjugated Ab's were incubated for $40 \mathrm{~min}$ at room temperature. Immunohistochemical staining was visualized using a laser-scanning confocal microscope (MRC 1024; Bio-Rad Laboratories Inc., Hercules, California, USA) using Lasersharp software version 2.1 (Bio-Rad Laboratories Inc.). Multicolor images were produced by overlaying several single-color images.

Chemotaxis assays. Chemotaxis assays were performed as described (20). Briefly, Transwell inserts (Corning Costar Corp., Cambridge, Massachusetts, USA) containing $2 \times 10^{6}$ tonsil lymphocytes were placed in the wells of 24-well plates in contact with $600 \mu \mathrm{l}$ of medium (RPMI-1640 with $0.5 \%$ BSA) with or without chemokine. After $2 \mathrm{~h}$, a fixed volume of counting beads (Polysciences Inc., Warrington, Pennsylvania, USA) was added to each well for normalization, and the specific migration was enumerated by flow cytometry after staining for the lymphocyte subsets of interest. Human TECK, MEC, and SDF-1 $\alpha / C X C L 12$ were purchased from Peprotech Inc. (Rocky Hill, New Jersey, USA).

Flow cytometry. Unconjugated mAbs were detected using either a biotinylated horse anti-mouse IgG secondary Ab (Vector Laboratories, Burlingame, California, USA) and streptavidin-peridinin chlorophyll protein (streptavidin-PerCP; PharMingen), or a goat anti-mouse-PE $\quad \mathrm{F}\left(\mathrm{ab}^{\prime}\right)_{2}$ secondary $\mathrm{Ab}$ (Jackson ImmunoResearch Laboratories Inc.). Four-color flow cytometry was done on a FACScalibur (Becton Dickinson Immunocytometry Systems, San Jose, California, USA) using CellQuest software, version 3.1 (Becton Dickinson Immunocytometry Systems).

\section{Results}

To identify tissue lymphocyte subsets expressing the chemokine receptor CCR10, we first examined memory a
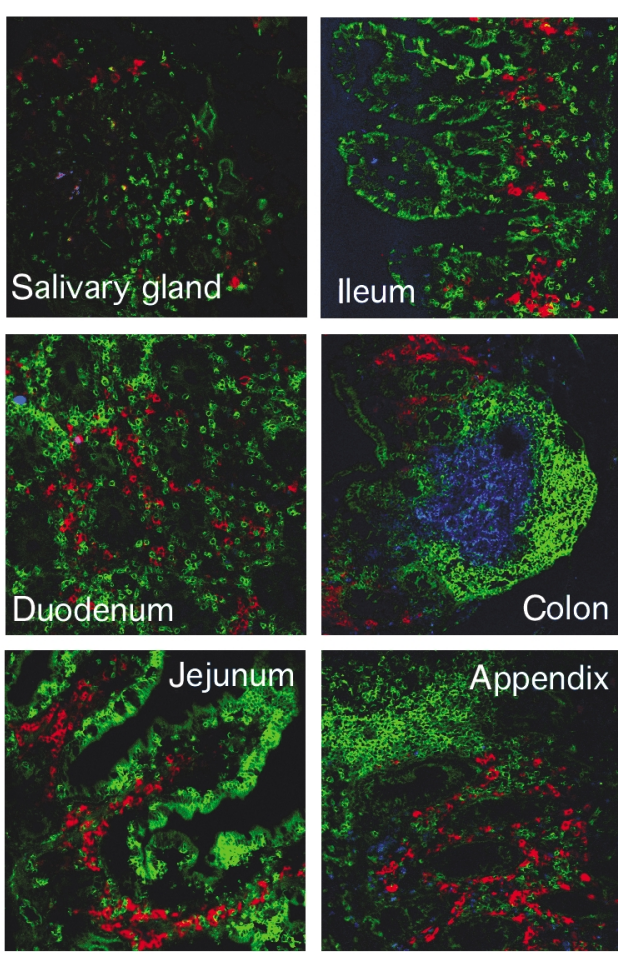

$\mathrm{CD} 3 /$
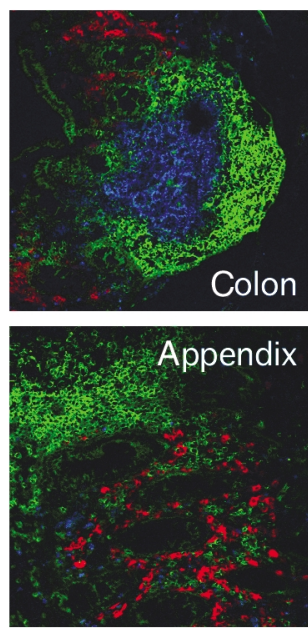

/CD20 b
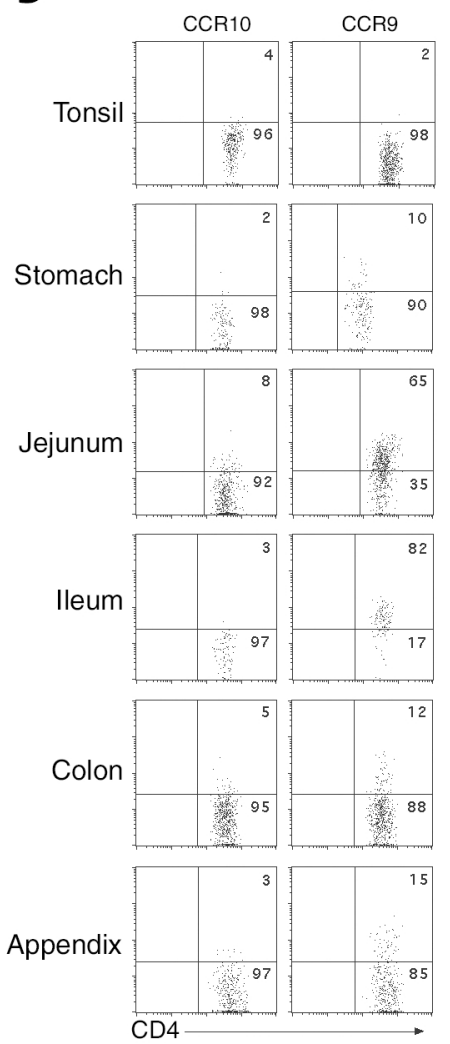

$\mathrm{CD}^{+}$memory
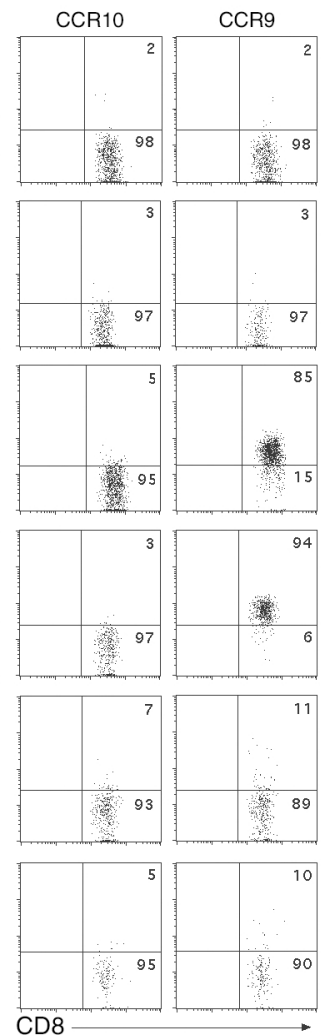

Figure 1

CCR10 is expressed on few gastrointestinal tissue Tlymphocytes. (a) Frozen sections of epithelial tissues were costained with Ab's against CD3 ( T cells; green), CCR10 (red), and CD20 (naive, memory, and GC B cells; blue). The lack of coexpression of CCR10 with CD3 or CD20 demonstrates that the cells expressing CCR10 within these tissues are not memory T or B cells. (b) T lymphocytes from various segments of the gastrointestinal tract and the tonsil were isolated and stained for $\mathrm{CD}^{+}$or $\mathrm{CD}^{+}$(and the marker CD45RA) in conjunction with CCR10 or CCR9. CCR10 is virtually absent on T cells within these tissues ( $<5 \%$ CCR $10^{+}$T cells in any examined tissue), while CCR9 is expressed on almost all T cells from the small intestine $(85 \% \pm 11 \%$ in the jejunum and $87 \% \pm 9 \%$ in the ileum), and a subpopulation of T cells in the colon (15\% $\pm 8 \%)$ and stomach $(11 \% \pm 6 \%)$, as described (14). Immunohistochemistry data is representative of three salivary gland, five jejunum, three ileum, two duodenum, four colon, and three appendix samples (and three tonsil and three stomach samples that are not shown). Flow-cytometry data are representative of two tonsil, three stomach, three jejunum, three ileum, three colon, and two appendix samples with mean \pm SD shown. Percentage of CCR10- or CCR9-positive cells based on quadrant encompassing 3\% of isotype control-stained cells in dot blots. 
$\mathbf{a}$
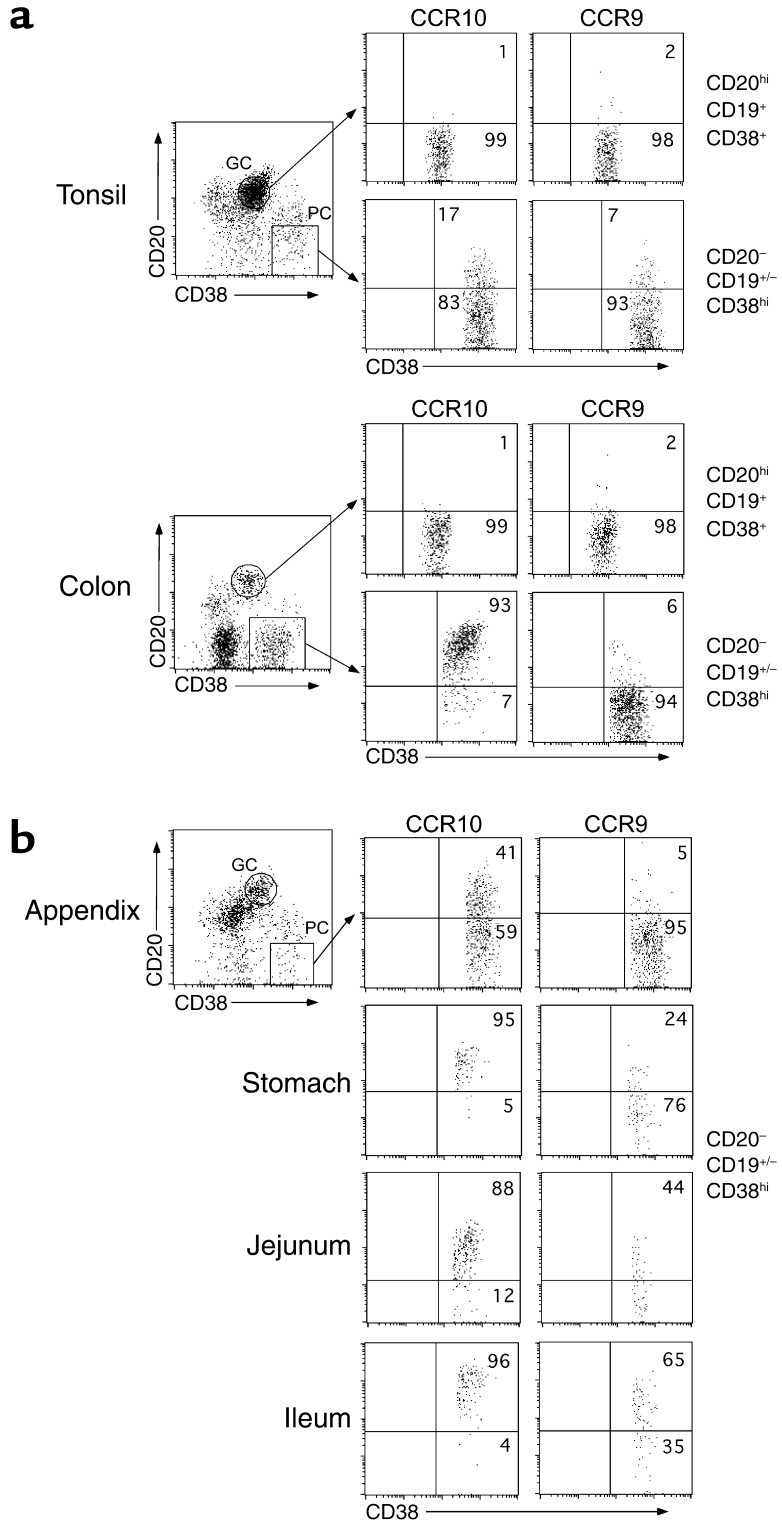

C

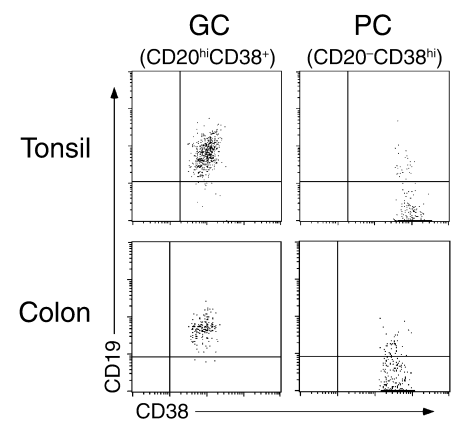

$\mathrm{T}$ lymphocytes in a variety of human epithelial tissues (Figure 1) where MEC is also expressed $(21,22)$. Surprisingly, flow cytometry (Figure $1 \mathrm{~b}$ ) revealed that CCR10 was not expressed on very many epithelial tissue memory $\mathrm{T}$ lymphocytes, although the small intestine and, to a lesser extent, the colon and stomach, contained CCR9 ${ }^{+}$lymphocytes as shown previously $(14,15)$.

\section{Figure 2}

CCR10 is expressed on mucosal tissue lymphocytes with a PC phenotype. Lymphocytes isolated from the gastrointestinal tract or tonsil were stained for CD19, CD20, and CD38 to define various B cell subsets (gated on large lymphocytes by scatter). (a) Both the colon and tonsil contained significant populations of both GC $\left(\mathrm{CD} 38^{+} \mathrm{CD} 19^{+} \mathrm{CD} 20^{\text {hi }}\right)$ and $\mathrm{PC}\left(\mathrm{CD} 38^{\text {hi }} \mathrm{CD} 19^{+/-} \mathrm{CD} 20^{-}\right)$phenotype $\mathrm{B}$ cells. GC cells did not express either CCR10 or CCR9 ( $<3 \%$ of GC cells in all tissues examined), while a fraction of PCs in the tonsil $(22 \% \pm 4 \%)$ and virtually all PCs in the colon $(92 \% \pm 4 \%)$ expressed CCR 10 . (b) CCR10 was also expressed on the vast majority of PCs in the jejunum $(90 \% \pm 7 \%)$, ileum $(92 \% \pm 5 \%)$, and stomach $(87 \% \pm 10 \%)$, and a significant fraction of cells in the appendix ( $38 \% \pm 11 \%)$. CCR9 was present on PCs in the jejunum ( $36 \% \pm 14 \%)$ and ileum $(41 \% \pm 18 \%)$ where TECK is also expressed and on PCs in the stomach (13\% $\pm 7 \%)$. (c) GC cells in both the tonsil and colon expressed high levels of CD19 (>95\% positive), while the phenotypically defined PC in both tissues had largely downregulated CD19 ( $<15 \%$ positive). Flow-cytometry data are representative of two tonsil, three stomach, three jejunum, three ileum, three colon, and two appendix samples with mean \pm SD shown. Percentage of CCR10- or CCR9-positive cells based on quadrant encompassing $5 \%$ of isotype control-stained cells in dot blots.

Neither freshly isolated T lymphocytes nor cells incubated at $37^{\circ} \mathrm{C}$ for $1 \mathrm{~h}$ stained detectably with antiCCR10 mAb's. Additionally, few, if any, memory T lymphocytes expressing CCR10 could be detected in the tonsil or appendix. Immunohistochemistry of various mucosal epithelial tissues also failed to reveal CCR10 expression on tissue memory T cells (Figure 1a). Thus, CCR10 expression on Tlymphocytes appears to be characteristic of CLA ${ }^{+}$skin-homing memory lymphocytes $(18,19)$, but not of the major mucosal memory T cell populations in these tissues.

In mice we have found that TECK, an epithelial chemokine ligand for CCR9 (15), is chemotactic for intestinal IgA Ab-secreting cells (16). Since TECK is closely related to $\operatorname{MEC}(21,22)$, we decided to examine the expression of CCR10 on the major B cell population resident in mucosal epithelial tissues: the plasma cell (Figure 2). In humans, markers for differentiating $B$ cells are well characterized $(25,26)$. Germinal center (GC) B cells can be defined as large $\mathrm{CD} 38^{+} \mathrm{CD} 19^{+} \mathrm{CD} 20^{\mathrm{hi}}$; plasma cells (PCs) are defined as large $\mathrm{CD} 38^{\mathrm{hi}} \mathrm{CD} 19^{+/-} \mathrm{CD} 20^{-}$; and memory $\mathrm{B}$ cells are defined as small or large $\mathrm{CD} 38^{-} \mathrm{CD} 19^{+} \mathrm{CD} 20^{+}$. In the tonsil and colon, $\mathrm{CD} 38$ and CD20 expression defined populations of both GC cells and PCs in the blast population (Figure 2a). GC cells in both the tonsil and colon were negative for both CCR9 and CCR10, consistent with their nonmigratory nature. Interestingly, while a fraction of tonsil PC expressed CCR10 (and a smaller fraction expressed CCR9), colon PCs were virtually all CCR $10^{+}$. Similarly, $85-95 \%$ of PCs in the stomach, jejunum, and ileum expressed CCR10, while a smaller percentage of small-intestinal and some stomach PCs expressed CCR9 (Figure 2b). In the appendix, a hybrid lymphoepithelial tissue, about $40 \%$ of the PC phenotype cells expressed CCR10 (Figure 2b), but no memory $\mathrm{B}$ cells present in any tissue (even the tonsil or appendix) expressed CCR10 (data not shown). Less than 
$15 \%$ of the CCR $10^{+}$PCs in both lymphoid and nonlymphoid tissues expressed CD19, suggesting that the majority of these PCs are terminally differentiated (Figure 2c). Thus, expression of CCR 10 on PCs in the stomach, small intestine, and colon correlates with the expression of MEC in these tissues $(21,22)$, while expression of CCR9 on small-intestinal PCs correlates with restricted small intestinal TECK expression (14).

IgA is the predominant immunoglobulin produced by resident PCs in the mucosal epithelial tissues examined here (3), so we used immunohistochemistry to examine the expression of CCR10 by mucosal $\mathrm{B}$ cells expressing IgA (Figure 3). In the colon, CCR10 expression colocalized to cells with IgA expression (Figure 3a). A similar colocalization of CCR10 with IgA was seen in the other mucosal epithelial tissues we examined (Figure $3 \mathrm{~b})$. A small percentage $(<5 \%)$ of PCs expressing IgG are also present in mucosal tissues (3), and CCR10 did not appear to colocalize with many of these cells by immunohistochemistry, although several $\mathrm{IgG}^{+}$cells were faintly CCR $10^{+}$(Figure $3 \mathrm{c}$ ), similar to the staining profile of circulating IgG plasmablasts (see Figure 5 below). IgG Ab-secreting cells in inflamed rheumatoid synovium did not express CCR10 (data not shown), suggesting that CCR10 may characterize mucosal-associated IgG Absecreting cells. Taken together, these results identify mucosal IgA PCs as the predominant (non-skin homing) lymphocyte subset expressing CCR10.

The development of IgA Ab-secreting cells occurs primarily in lymphoid tissues (27) (although recent data has suggested that in situ class switching to IgA may also occur within the murine small intestine; see ref. 28). Early IgA PCs, or plasmablasts, travel from lymphoid tissues through the blood to mucosal epithelial tissues, where they localize and secrete IgA. To determine if CCR10 could be involved in plasmablast trafficking, we asked if differentiating tonsil surface $\operatorname{IgA} \mathrm{A}^{+}\left(\operatorname{sIgA}^{+}\right) \mathrm{B}$ cells could migrate to MEC (Figure 4a). Interestingly, tonsil B cells with a plasmablast phenotype (large sIgA $\left.{ }^{+} \mathrm{CD} 38^{\text {hi }} \mathrm{CD} 19^{+} \mathrm{CD} 20^{-}\right)$migrated to MEC, while MEC responsiveness was reduced in phenotypically defined mature PCs (large sIgA ${ }^{+/} \mathrm{CD} 38^{\mathrm{hi}} \mathrm{CD} 19^{+/}{ }^{-} \mathrm{CD} 20^{-}$, the phenotype of effector tissue PCs). Little chemotaxis to TECK of either population was observed (Figure 4a). The ability of tonsil PCs to migrate to MEC, but only weakly to TECK, correlated clearly with the high percentage of these PCs expressing CCR10 (particularly $\mathrm{IgA}^{+}$cells) but not CCR9 (Figure 4b).

We next examined circulating lymphocytes to determine if phenotypically defined IgA plasmablasts expressing CCR10 were present (Figure 5). While circulating small IgA memory B cells (Figure 5a; small $\left.\operatorname{sIgA}^{+} \mathrm{CD} 19^{+}\right)$did not express CCR10, a subset expressed
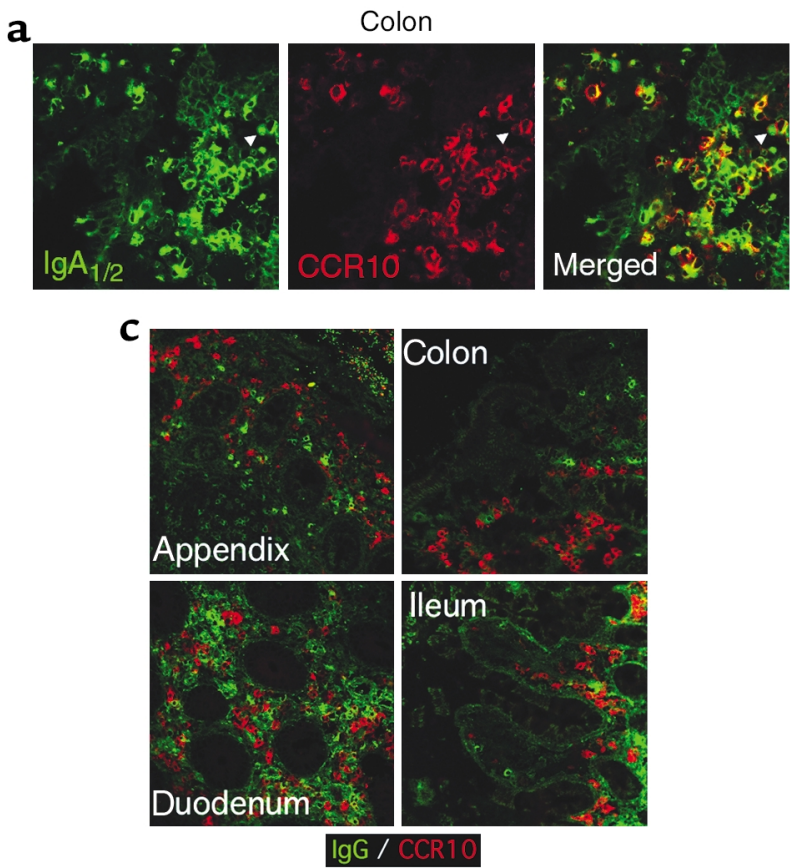
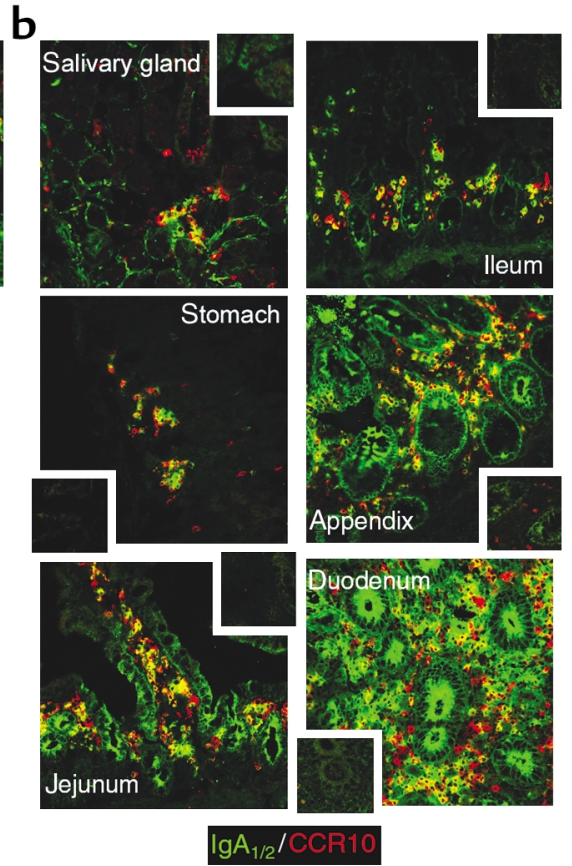

\begin{abstract}
Figure 3
CCR10 is expressed by mucosal tissue IgA plasma cells. Epithelial tissues known to be sites of IgA plasma cell localization were costained for CCR10 (red) and $\lg A_{1 / 2}$ (green; in $\mathbf{a}$ and $\mathbf{b}$ ) or IgG (green; panel $\mathbf{c}$ ). (a) The expression pattern of IgA on colon lymphocytes is identical to that of CCR10. Because CCR10 is surface expressed and IgA can be both on the surface and intracellular, the merged panel includes regions of yellow overlap as well as green and red areas. (b) Merged images of CCR10 and IgA staining from other tissues containing IgA plasma cells demonstrate that CCR10 colocalizes with IgA in all sites examined. Luminally transported secretory IgA can be seen in the lumens of the duodenum, jejunum, and appendix. Insets show negative control staining. (c) A small fraction of IgG PCs are present in mucosal tissues, but CCR10 did not colocalize well with these cells. Immunohistochemistry data is representative of three salivary gland, three stomach, five jejunum, three ileum, two duodenum, five colon, and three appendix samples.
\end{abstract}



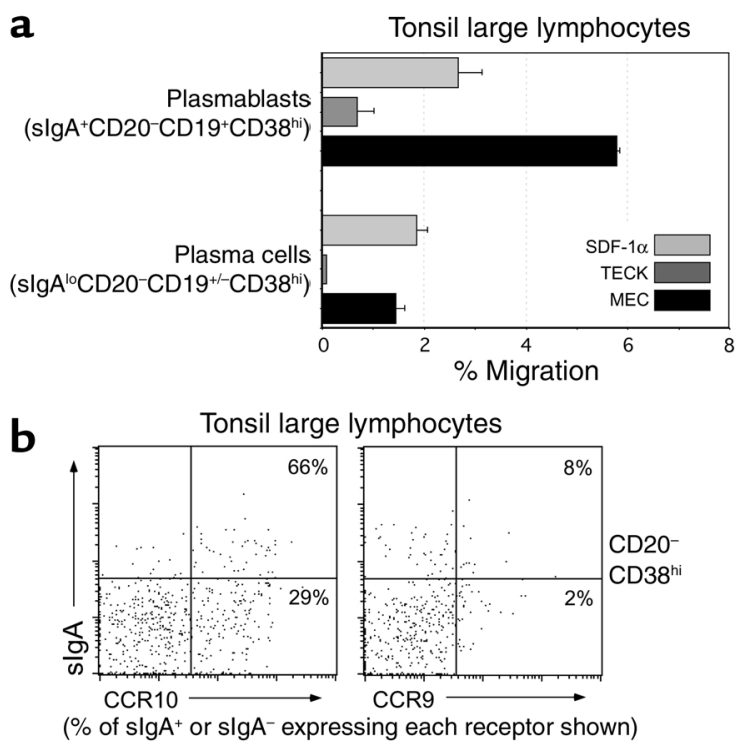

\section{Figure 4}

MEC is chemotactic for tonsil IgA plasmablasts expressing CCR10. (a) Tonsil lymphocytes were migrated to medium, SDF-1 $\alpha(100$ $\mathrm{nM})$, TECK (300 nM), or MEC (300 nM) and stained for sIgA, CD19, CD20, and CD38 to identify plasmablasts and plasma cells in the large lymphocyte gate. Only slgA ${ }^{+} \mathrm{B}$ cells with a plasmablast phenotype migrated well to MEC. Plasmablasts did not migrate well to TECK. (b) Consistent with their migration to MEC, tonsil plasmablasts and plasma cells (CD38 ${ }^{\text {hi }}$ CD $\left.20^{-}\right)$express CCR10 $\left(60 \% \pm 14 \%\right.$ of $s / g A^{+}$plasmablasts and $21 \% \pm 13 \%$ of $\left.\operatorname{slg} A^{\mathrm{lo} /-} \mathrm{PCs}\right)$, while the lack of a TECK response is consistent with the small number of cells expressing CCR9 $\left(10 \% \pm 6 \%\right.$ of slgA $A^{+}$plasmablasts and $4 \% \pm 2 \%$ of slgA $A^{\mathrm{lo} /-} \mathrm{PC}$ ) . Data are representative of two independent experiments with multiple wells per experiment (the percentage of cells expressing CCR10 or CCR9 is averaged over both experiments, mean $\pm S D$ ). As stated, the percentages shown in $\mathbf{b}$ are the percentages of $\operatorname{sig} \mathrm{A}^{+}$or $\mathrm{s} / \mathrm{g} \mathrm{A}^{-}$plasma cells expressing each receptor. Background migration was $<1 \%$ in both cell populations.

CCR9, and virtually all were $\mathrm{CCR} 6^{+}$; a similar receptor expression profile was seen on $\operatorname{sig} \mathrm{A}^{+} \mathrm{CD} 19^{+}$large $\mathrm{B}$ cell blasts (Figure $5 \mathrm{~b}$ ). In fact, the only identifiable blood $\mathrm{B}$ cell population to express CCR10 was $\operatorname{sgA}{ }^{+} \mathrm{B}$ cells with a plasmablast phenotype (large $\left.\operatorname{sIg} A^{\text {int }} C D 19^{\text {int }}\right)$, and the majority of these IgA $\mathrm{A}^{+}$plasmablasts were CCR $10^{+}$(Figure $5 \mathrm{~b}$; range $=62-80 \%$, mean $\pm \mathrm{SD}=72 \% \pm 8 \%$ ). A subset of these IgA plasmablasts also expressed CCR9, and interestingly, as described during in vitro differentiation of human B cells into PCs (29), CCR6 expression was concomitantly reduced (CCR6 was similarly expressed on only approximately $15 \%$ of tissue PCs; data not shown). Confirming our immunohistochemical observation that CCR10 expression was low to absent on mucosal tissue IgG Ab-secreting cells, few circulating $\operatorname{IgG}$ plasmablasts (large $\operatorname{sIg} G^{\text {int }} \mathrm{CD} 19^{\text {int }}$ ) expressed CCR10 or CCR9 (positive cells ranged from $8 \%$ to $20 \%$ for both receptors, depending on the donor, and the staining was generally of low intensity across donors), and these cells had also downregulated CCR6 (Figure 5c). Thus, the presence of migratory MECresponsive CCR $10^{+}$tonsil plasmablasts coupled with the predominant expression of CCR10 on circulating $\operatorname{IgA}{ }^{+}$plasmablasts strongly suggests a role for CCR10 in IgA plasmablast trafficking to and migration into effector tissues expressing MEC. Similarly, IgA plasmablasts expressing CCR9 may localize to the small intestine (where TECK is expressed) after arising in intestinal lymphoid tissues.

\section{Discussion}

We show here that CCR10 expression characterizes epithelial tissue IgA PCs in tissues where MEC is expressed, but not memory $\mathrm{T}$ or $\mathrm{B}$ cells and few IgG PCs. The luminal transport of secretory $\operatorname{IgA}$ is an important part of host protection at epithelial surfaces (1-3). The presence of MEC in gastrointestinal tissues, the salivary gland, mammary gland, and trachea $(21$, 22) may provide a mechanism for circulating CCR $10^{+}$ IgA plasmablasts to enter all of these diverse tissues, while conversely, CCR9 expression on a subset of circulating IgA plasmablasts may facilitate and potentially focus their localization to the small intestine (see Figure 6). It is worth noting that the roles of MEC and TECK in IgA Ab-secreting cell biology are well conserved between human and mouse: we find that in mice, MEC is a highly efficacious chemoattractant for $\operatorname{IgA}$ (but not $\operatorname{IgG}$ or $\operatorname{IgM}$ ) Ab-secreting cells from multiple mucosal tissues (small and large intestines, lung, and mammary gland) and the lymph nodes that drain those tissues, while TECK responsiveness is relatively limited to IgA Ab-secreting cells within the small intestine and its associated lymphoid tissues (ref. 16; N.H. Lazarus and E.J. Kunkel, unpublished data).

Although the IgA immune system has historically been considered a "common mucosal immune system" (7), more recent data suggest that the IgA immune system has both common and regional characteristics. Small intestine-derived IgA plasmablasts appear to be more likely to migrate to both the intestines and nonintestinal tissues, such as the upper airways, lacrimal, salivary, and mammary glands $(6,8)$, suggesting some commonality. However, the restricted transfer of antigen-specific IgA to the upper airways, lacrimal gland, nasal cavity, and genital tract after nasal immunization $(5,8)$, suggests regionalization of the upper aero-digestive and genital tract. A role for CCR10 and MEC/CCL28 in IgA Ab-secreting cell localization to both intestinal and nonintestinal mucosal sites is compatible with both common and regional characteristics. This is because, in addition to chemokines, immune cell trafficking from the blood into tissues requires one or more pairs of adhesion molecules (30). IgA plasmablasts and PCs within the intestines are almost uniformly positive for the $\alpha_{4} \beta_{7}$ integrin (31), consistent with the expression of the $\alpha_{4} \beta_{7}$ integrin ligand MAdCAM-1 on intestinal endothelium (32). In the mouse, mammary gland IgA plasmablasts also express $\alpha_{4} \beta_{7}$ integrin, and lactating mammary gland endothelium expresses MAdCAM-1 (33). Interestingly, salivary gland endothelium does not express MAdCAM-1, but it does 
express VCAM-1 (34), and the ability of $\alpha_{4} \beta_{1}$ and possibly $\alpha_{4} \beta_{7}$ on IgA plasmablasts to bind VCAM-1 (35) may facilitate their migration into the salivary gland and other epithelial tissues such as the trachea and bronchioles (36). Thus, IgA plasmablasts arising in intestinal lymphoid tissues that coexpress CCR10 and $\alpha_{4} \beta_{7}$ may be uniquely able to enter MEC-containing mucosal sites where either MAdCAM- 1 or VCAM- 1 are expressed, while those intestinal IgA plasmablasts that also express CCR9 can additionally enter the small intestine where TECK is expressed. Conversely, IgA plasmablasts arising in nonintestinal mucosal lymphoid tissues such as the tonsils and adenoids that largely lack CCR9 (and express lower levels of $\alpha_{4} \beta_{7}$; E.J. Kunkel, unpublished data) may therefore be restricted to nonintestinal mucosal sites where they use CCR10 in conjunction with other adhesion molecules such as $\alpha_{4} \beta_{1}$ and L-selectin (8).

CCR9 and CCR10 exhibit interesting expression patterns that appear to depend on both differentiation state and potentially the site of antigen exposure. While neither CCR9 (15) nor CCR10 appear to be expressed on resting naive $\mathrm{B}$ cells, we cannot rule out that they are expressed at very low levels. CCR9 is expressed on approximately $20 \%$ of circulating $\operatorname{IgA}^{+}$B cells with a memory phenotype. In contrast, CCR 10 is absent from memory B cells but is expressed on IgA class-switched plasmablasts and PCs. Therefore, the expression of both receptors may be regulated by cytokines such as
TGF- $\beta$ that are involved in IgA class switching (37). The absence of CCR10 on memory B cells, however, suggests that CCR10 expression may be more closely linked to PC differentiation, particularly in mucosal sites. The expression of CCR9 on a subset of memory B cells may mean that these $B$ cells were originally activated in small-intestinal-associated lymphoid tissues, but the specific signals involved in CCR9 induction (e.g., TGF- $\beta$ and/or MAdCAM- $1^{+}$dendritic cells) remain to be elucidated. Our data characterizing smallintestinal PCs indicate that approximately $50 \%$ express both CCR9 and CCR10. This may mean that these double positive cells arose from $\operatorname{IgA}^{+}$memory $\mathrm{B}$ cells expressing CCR9 (or naive $\mathrm{B}$ cells activated in smallintestinal lymphoid tissues) and acquired CCR10 upon PC differentiation. Expression of both CCR9 and CCR10 (in conjunction with $\alpha_{4} \beta_{7}$ integrin) would allow these plasmablasts to enter mucosal tissues throughout the body. Conversely, $\operatorname{IgA}^{+}$memory $\mathrm{B}$ cells that do not express CCR9 may therefore represent B cells that class-switched in lymphoid tissues outside of the small intestine (for instance in large-intestinal follicles or the tonsil, where only $5 \%$ of the PCs express CCR9). These CCR9- $\mathrm{IgA}^{+}$memory cells would become CCR $10^{+}$upon PC differentiation and traffic into mucosal tissues expressing MEC, including the small intestine if they also coexpress $\alpha_{4} \beta_{7}$ integrin (a likely scenario for PCs induced in colon follicles but not in the tonsil). As more information about the genetic control of B cell

\section{Figure 5}

Subsets of circulating blood IgA plasmablasts express CCR10 and CCR9. Peripheral blood lymphocytes were isolated, depleted of T cells, and stained for surface IgA (slgA) or surface $\lg G(\operatorname{slg} G), C D 19$, a dump cocktail (CD14, CD3, IgD, and CD94), and CCR10, CCR9, or CCR6. (a) Of small memory lymphocytes expressing $\operatorname{sigA}\left(\mathrm{CD} 19^{+} \operatorname{slgA}^{+} ; 4 \% \pm 2 \%\right.$ of total CD19+ cells), few expressed CCR10 $(3 \% \pm 2 \%)$, while a small population expressed CCR9 $(16 \% \pm 5 \%)$, and virtually all expressed CCR6 $(98 \% \pm 1 \%)$. (b) Two discernable populations of dump cocktail-negative lymphocytes in the large lymphocyte gate could be identified by expression of sIgA and CD19. Large lymphocytes with an IgA memory phenotype $\left(\operatorname{sig} A^{+} \mathrm{CD} 19^{+}\right)$expressed CCR10 $(4 \% \pm 1 \%)$, CCR9 $(28 \% \pm 3 \%)$, and CCR6 $(90 \% \pm 2 \%)$ in a pattern similar to small slgA+ memory lymphocytes. A large fraction of lymphocytes with an IgA plasmablast phenotype

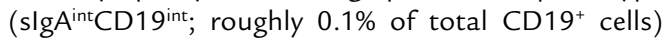
expressed CCR10 $(72 \% \pm 8 \%)$, and a smaller fraction expressed CCR9 $(17 \% \pm 13 \%)$, while few expressed CCR6 $(13 \% \pm 3 \%)$. (c) The majority $(>90 \%)$ of slgA CD19+/int lymphocytes (shown in b by box) are slgG ${ }^{+}$. $\mathrm{IgG}^{+}$memory lymphocytes $\left(\mathrm{slgG}^{+} \mathrm{CD} 19^{+}\right)$did not express CCR10 (3\% $\pm 2 \%$ ), while some expressed CCR9 $(14 \% \pm 3 \%)$, and almost all expressed CCR6 $(84 \% \pm 6 \%)$. Plasmablasts expressing slgG (slgG ${ }^{+}$CD19 $\left.19^{\text {int }}\right)$ contained some CCR $10^{+}(13 \% \pm 6 \%)$ and CCR9 ${ }^{+}(16 \% \pm 7 \%)$ lymphocytes and few CCR6 $6^{+}(18 \% \pm 4 \%)$ cells. Data are representative of four experiments from separate blood donors with data averaged across donors (mean $\pm \mathrm{SD}$ ).

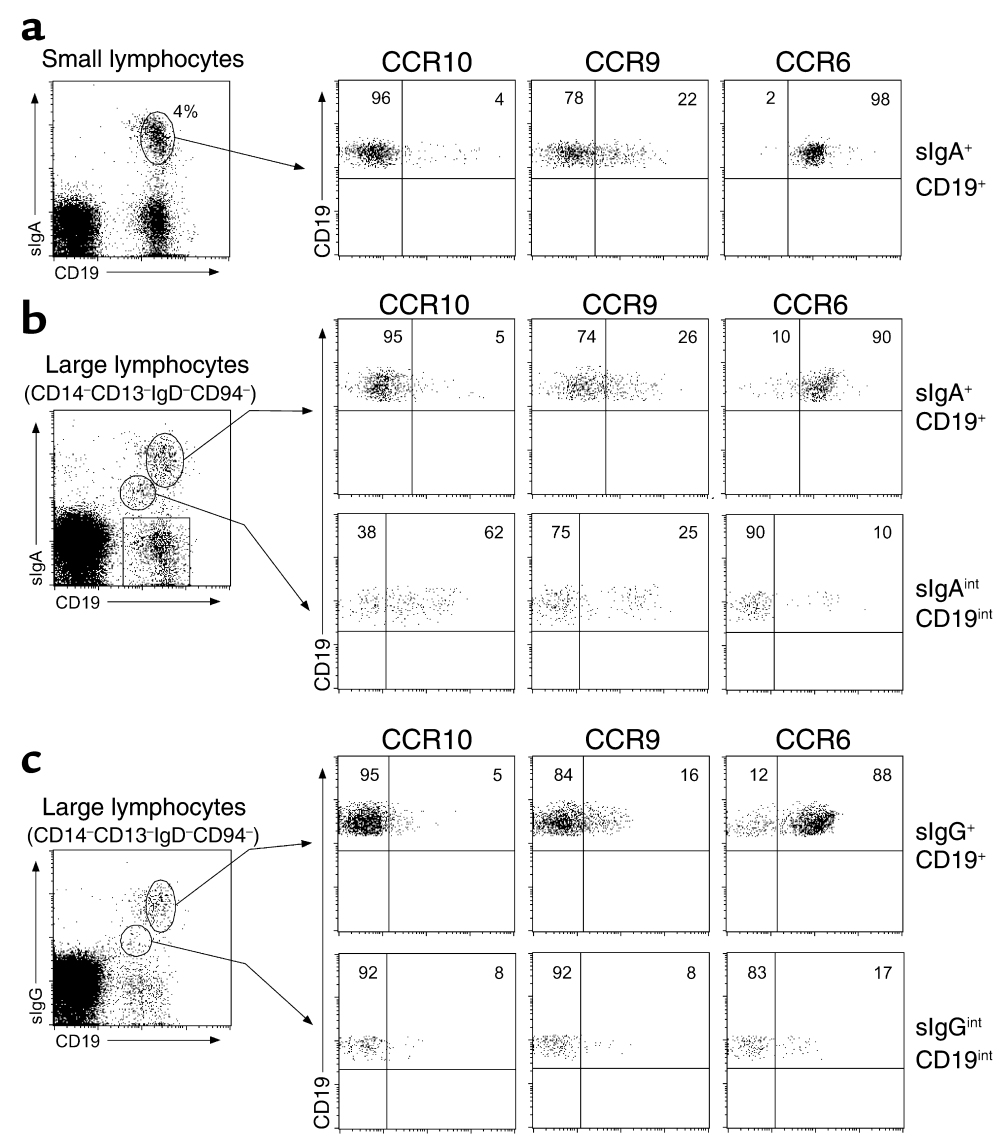




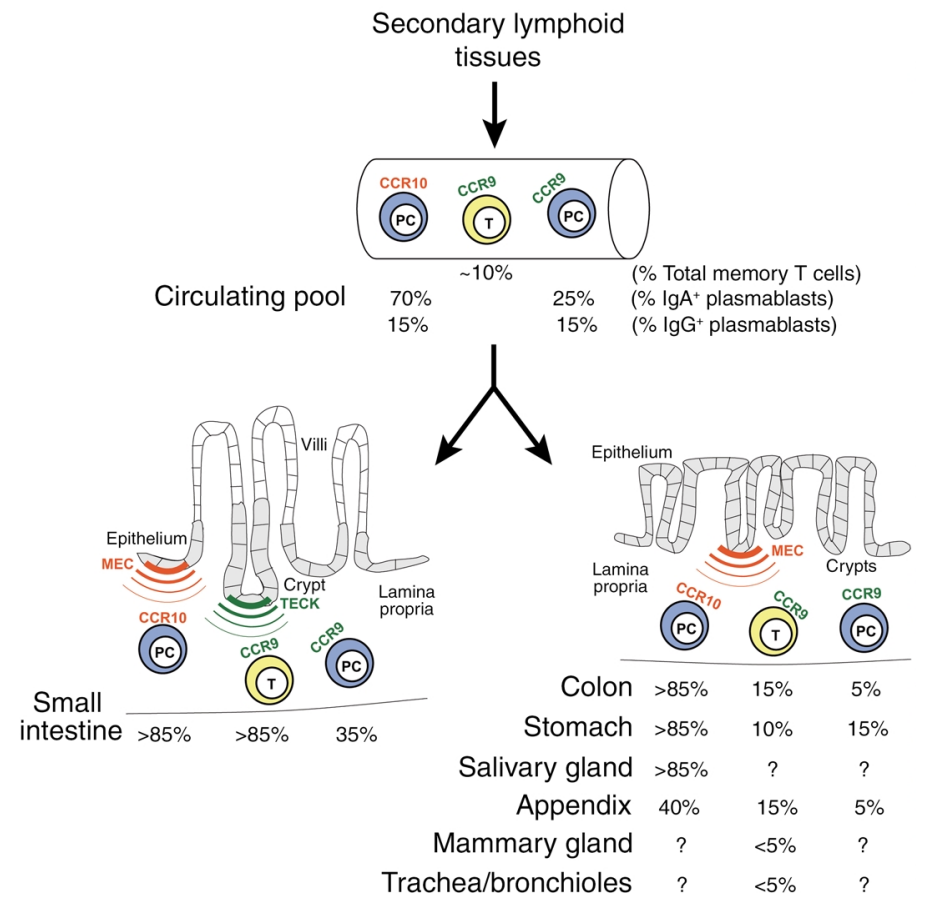

\section{Figure 6}

MEC and CCR10 unify the epithelial IgA immune system. After development in secondary lymphoid tissues (i.e., the tonsil or appendix), IgA plasma cells (PCs) expressing CCR10 or CCR9 enter the circulation. By virtue of the expression of both MEC and TECK in the small intestine, both CCR $10^{+}$ and CCR9 ${ }^{+}$PCs can enter this tissue (although CCR10 ${ }^{+}$PCs predominate). In other tissues where MEC expression predominates (i.e., the colon and stomach), CCR10+ PCs predominate, and CCR9 ${ }^{+} \mathrm{PCs}$ are more rare. Other epithelial sites where MEC is expressed (i.e., the mammary gland and trachea/bronchioles) and IgA is secreted may also contain CCR $10^{+}$PCs. CCR9 ${ }^{+} \mathrm{T}$ cells $(\mathrm{T})$ predominate in the small intestine where TECK is expressed and exist at lower levels in closely associated tissues such as the stomach and colon. This separate, previously described, T lymphocyte localization pathway for the small intestine (via CCR9/TECK) allows functional compartmentalization of this organ, while physically dispersed organs that all share the function of pathogen neutralization by IgA are unified by CCR10/MEC. Model includes data from refs. 14, 21, 22. class switching and PC differentiation is uncovered (38), it will be possible to determine what specific inductive signals control the expression of both CCR9 and CCR10 (and specific integrins such as $\alpha_{4} \beta_{7}$ ) during $B$ cell differentiation.

Our results provide an explanation for the expression of MEC within a variety of mucosal epithelial tissues, even though the only $\mathrm{T}$ lymphocyte subset previously (or in our study) shown to express CCR10 (CLA ${ }^{+}$skin-homing lymphocytes) does not enter these sites $(18,19,23)$. The evolutionary rationale behind the use of one chemokine receptor (CCR10) for the homeostatic localization of two very distinct leukocyte subsets (memory T cells and IgA PCs) is intriguing. The close homology between the homeostatically expressed epithelial chemokines MEC, TECK, CTACK and the inducible epithelial chemokine MIP-3 $\alpha / C C L 20(21,22)$ suggests that they evolved from a common ancestor and then became associated with one or more epithelial cell populations as the need arose for specific types of host protection at diverse epithelial sites. In general, the adhesion receptors controlling lymphocyte recruitment into the skin and intestines have become dissimilar enough that CCR10 can be used selectively on both CLA ${ }^{+} \mathrm{T}$ cells and IgA plasmablasts, particularly within the framework of the combinatorial control of lymphocyte trafficking and localization (30). $\mathrm{CLA}^{+}$skin-homing $\mathrm{T}$ cells are virtually never found within the intestinal tract, likely because they lack the gut homing receptor $\alpha_{4} \beta_{7}$ necessary for interaction with MAdCAM- $1^{+}$intestinal venules (23) and because intestinal venules lack expression of the adhesion-triggering CCR4 ligand TARC/CCL17 (20). So even though CLA Cymphocytes $^{+}$ expressing CCR10 can respond to MEC, their entry into MEC-expressing gastrointestinal sites would be extremely inefficient. CLA ${ }^{+}$T cells do express the $\alpha_{4} \beta_{1}$ integrin required to bind VCAM-1 expressed by endothelia in mucosal sites such as the oral cavity, salivary gland, and lung $(23,34,36)$. Nevertheless, although some CLA ${ }^{+} \mathrm{T}$ cells can be found in the oral mucosa and lung (12), it is unknown whether CCR10 and MEC play a role in this localization.

Our results also demonstrate an interesting bifurcation in the evolutionary development of tissue-specific $\mathrm{T}$ and $\mathrm{B}$ cell trafficking. CCR9 appears to be rather specifically associated with both small-intestinal IgA Ab-secreting cells (consistent with our previous finding in the murine system; ref. 16) and small-intestinal $\mathrm{T}$ cells $(14,17)$, while it is largely absent from both $\mathrm{T}$ and PCs in other mucosal sites. On the other hand, even though the CCR10 ligand MEC is expressed in a variety of mucosal tissues (21), few if any T cells in these tissues express CCR10. Thus, in contrast to the small intestine where CCR9 appears to be involved in both $\mathrm{T}$ and B cell biology, CCR10 is associated with mucosal PC trafficking and cutaneous T cell trafficking $(19,39)$. More investigation is required to determine the reasons for the evolutionary segregation of the small intestine through CCR9 and the differential use of CCR10 for mucosal B cell, but not $\mathrm{T}$ cell, trafficking.

In addition to expressing CCR9 and CCR10, as we show here, circulating and tissue-resident IgA (and IgG) plasmablasts appeared to downregulate their expression of CCR6, the receptor for MIP-3 $\alpha / C C L 20$ (40). In addition to being expressed on subsets of memory $\mathrm{T}$ cells (41), CCR6 is also expressed on memory and naive B cells, and, as we confirm in vivo, is downregulated as B 
cells mature into PCs in vitro (29). Our findings would suggest that CCR6 is likely not involved in IgA plasmablast localization to epithelial tissues.

While mucosal epithelial tissues contain IgG PCs, these cells are usually less than $3-5 \%$ of the total PC population (3). Many more IgG PCs are found in the spleen and bone marrow (42). We find that few IgG PCs within MEC-expressing epithelial tissues express CCR10, and those that do appear to express lower levels. Similarly, in the blood, the CD19int large lymphocytes that express sIgG also contain few CCR $10^{+}$ cells. In the murine system, IgG Ab-secreting cells maintain high responsiveness to the chemokine SDF-1 $\alpha(16,43)$, while additionally becoming responsive to the CXCR3 ligand MIG/CXCL9 (16). The ability of murine IgG antibody secreting cells (ASC) to respond to SDF-1 $\alpha$ appears to play a role in localization to the bone marrow (43), while responsiveness to MIG may promote migration into inflammatory sites such as rheumatoid synovium. Few murine IgG ASCs (even from Peyer's patches or mesenteric lymph nodes, two intestinal-associated lymphoid tissues) respond to TECK (16) or MEC (N.H. Lazarus and E.J. Kunkel, unpublished data). Thus, it seems likely that, rather than homeostatic chemokines, inflammatory chemokines (particularly CXCR3 ligands) may play a dominant role in the localization of circulating $\operatorname{IgG}$ plasmablasts to epithelial (and other) tissues, allowing IgG plasmablast recruitment during tissue inflammation.

Interestingly, in patients with selective IgA deficiency, the mucosal compartments of the upper aerodigestive tract commonly contain large numbers of $\mathrm{IgD}^{+}, \mathrm{IgM}^{+}$, and $\mathrm{IgG}^{+}$PCs, while the intestinal mucosal compartments largely contain $\mathrm{IgG}^{+}$and $\mathrm{IgM}^{+} \mathrm{PCs}$ (3). It is unclear at this point whether CCR10 and MEC/CCL28 are involved in the mucosal migration of plasmablasts expressing other Ig isotypes, but in the absence of CCR10+ IgA plasmablasts, it is very likely that CCR $10^{+} \operatorname{IgG}$ and IgM Ab-secreting cells (which are only a small fraction of the total IgG and IgM plasmablast population in normal individuals) would have little competition for mucosal compartments and would therefore be able to fill these compartments. In fact, we have preliminary evidence that early in the human anti-rotavirus humoral response, rotavirus-specific plasmablasts expressing IgM or IgG can express CCR9 and/or CCR10 (M.C. Jaimes and E.J. Kunkel, unpublished data).

In conclusion, we find that CCR10 expression characterizes circulating and tissue plasmablasts and PCs that secrete the immunoglobulin IgA. We propose that CCR10, in conjunction with mucosal epithelial cell-derived MEC, contributes to the selective localization of IgA Ab-secreting cells to the physically dispersed organs of the secretory IgA immune system. Our findings provide a common chemoattractant mechanism for the dissemination of IgA-dependent immunity following local mucosal stimulation or vaccination.

\section{Acknowledgments}

We thank E. Resurreccion for excellent technical skill with the histology and K.R. Youngman and L.S. Rott for critical reading of this manuscript. E.J. Kunkel is an Arthritis Foundation postdoctoral fellow. C.H. Kim is a Leukemia and Lymphoma Society postdoctoral fellow. This work is supported by NIH grants GM-37734 and AI-47822 to E.C. Butcher, Specialized Centers of Research grant HL-67674, Digestive Disease Center grant DK-56339, and a Merit Award from the Veterans Administration to E.C. Butcher.

1. Kato, H., Kato, R., Fujihashi, K., and McGhee, J.R. 2001. Role of mucosal antibodies in viral infections. Curr. Top. Microbiol. Immunol. 260:201-228.

2. Offit, P.A. 2001. Correlates of protection against rotavirus infection and disease. Novartis. Found. Symp. 238:106-113.

3. Brandtzaeg, P., et al. 1999. The B-cell system of human mucosae and exocrine glands. Immunol. Rev. 171:45-87.

4. Czerkinsky, C., Svennerholm, A.M., Quiding, M., Jonsson, R., and Holmgren, J. 1991. Antibody-producing cells in peripheral blood and salivary glands after oral cholera vaccination of humans. Infect. Immun. 59:996-1001.

5. Johansson, E.L., Wassen, L., Holmgren, J., Jertborn, M., and Rudin, A. 2001. Nasal and vaginal vaccinations have differential effects on antibody responses in vaginal and cervical secretions in humans. Infect. Immun. 69:7481-7486.

6. Weisz-Carrington, P., Roux, M.E., McWilliams, M., Phillips-Quagliata, J.M., and Lamm, M.E. 1979. Organ and isotype distribution of plasma cells producing specific antibody after oral immunization: evidence for a generalized secretory immune system. J. Immunol. 123:1705-1708.

7. McDermott, M.R., and Bienenstock, J. 1979. Evidence for a common mucosal immunologic system. I. Migration of B immunoblasts into intestinal, respiratory, and genital tissues. J. Immunol. 122:1892-1898.

8. Brandtzaeg, P., Farstad, I.N., and Haraldsen, G. 1999. Regional specialization in the mucosal immune system: primed cells do not always home along the same track. Immunol. Today. 20:267-277.

9. van Ginkel, F.W., Nguyen, H.H., and McGhee, J.R. 2000. Vaccines for mucosal immunity to combat emerging infectious diseases. Emerg. Infect. Dis. 6:123-132.

10. Mestecky, J. 1987. The common mucosal immune system and current strategies for induction of immune responses in external secretions. J. Clin. Immunol. 7:265-276.

11. Russell, M.W., Hedges, S.R., Wu, H.Y., Hook, E.W., and Mestecky, J. 1999. Mucosal immunity in the genital tract: prospects for vaccines against sexually transmitted diseases-a review. Am. J. Reprod. Immunol. 42:58-63.

12. Kunkel, E.J., and Butcher, E.C. 2002. Chemokines and the tissue-specific migration of lymphocytes. Immunity. 16:1-4.

13. Wurbel, M.-A., et al. 2000. The chemokine TECK is expressed by thymic and intestinal epithelial cells and attracts double- and single-positive thymocytes expressing the TECK receptor CCR9. Eur. J. Immunol. 30:262-271.

14. Kunkel, E.J., et al. 2000. Lymphocyte CC chemokine receptor 9 and epithelial thymus-expressed chemokine (TECK) expression distinguish the small intestinal immune compartment: epithelial expression of tissue-specific chemokines as an organizing principle in regional immunity. J. Exp. Med. 192:761-768.

15. Zabel, B.A., et al. 1999. Human G protein-coupled receptor GPR-9-6/CC chemokine receptor 9 is selectively expressed on intestinal homing $\mathrm{T}$ lymphocytes, mucosal lymphocytes, and thymocytes and is required for thymus-expressed chemokine-mediated chemotaxis. J. Exp. Med. 190:1241-1255.

16. Bowman, E.P., et al. 2002. The intestinal chemokine TECK (CCL25) attracts IgA antibody secreting cells. J. Exp. Med. 195:269-275.

17. Papadakis, K.A., et al. 2000. The role of thymus-expressed chemokine and its receptor CCR9 on lymphocytes in the regional specialization of the mucosal immune system. J. Immunol. 165:5069-5076.

18. Morales, J., et al. 1999. CTACK, a skin-associated chemokine that preferentially attracts skin-homing memory T cells. Proc. Natl. Acad. Sci. U. S. A. 96:14470-14475.

19. Hudak, S., et al. 2002. Immune surveillance and effector functions of CCR10+ skin homing T cells. J. Immunol. 169:1189-1196.

20. Campbell, J.J., et al. 1999. The chemokine receptor CCR4 in vascular recognition by cutaneous but not intestinal memory T cells. Nature. 400:776-780.

21. Wang, W., et al. 2000. Identification of a novel CC chemokine (CCL28) which binds CCR10 (GPR2). J. Biol. Chem. 275:22313-22323. 
22. Pan, J., et al. 2000. A novel chemokine ligand for CCR10 and CCR3 expressed by epithelial cells in mucosal tissues. J. Immunol. 165:2943-2949.

23. Butcher, E.C., Williams, M., Youngman, K., Rott, L., and Briskin, M. 1999. Lymphocyte trafficking and regional immunity. Adv. Immunol. 72:209-253.

24. Jarmin, D.I., et al. 2000. Cutting edge: identification of the orphan receptor G-protein-coupled receptor 2 as CCR10, a specific receptor for the chemokine ESkine. J. Immunol. 164:3460-3464.

25. Farstad, I.N., Carlsen, H., Morton, H.C., and Brandtzaeg, P. 2000 Immunoglobulin A cell distribution in the human small intestine: phenotypic and functional characteristics. Immunology. 101:354-363.

26. Banchereau, J., and Rousset, F. 1992. Human B lymphocytes: phenotype, proliferation, and differentiation. Adv. Immunol. 52:125-262.

27. Brandtzaeg, P., et al. 1999. Regional specialization in the mucosal immune system: what happens in the microcompartments? Immunol. Today. 20:141-151.

28. Fagarasan, S., Kinoshita, K., Muramatsu, M., Ikuta, K., and Honjo, T. 2001. In situ class switching and differentiation to IgA-producing cells in the gut lamina propria. Nature. 413:639-643.

29. Krzysiek, R., et al. 2000. Regulation of CCR6 chemokine receptor expression and responsiveness to macrophage inflammatory protein3alpha/CCL20 in human B cells. Blood. 96:2338-2345.

30. Butcher, E.C. 1991. Leukocyte-endothelial cell recognition: three (or more) steps to specificity and diversity. Cell. 67:1033-1036.

31. Farstad, I.N., et al. 1995. Human intestinal B-cell blasts and plasma cells express the mucosal homing receptor integrin alpha 4 beta 7. Scand. J. Immunol. 42:662-672.

32. Briskin, M., et al. 1997. Human mucosal addressin cell adhesion molecule-1 is preferentially expressed in intestinal tract and associated lymphoid tissue. Am. J. Pathol. 151:97-110.

33. Tanneau, G.M., Hibrand-Saint, O.L., Chevaleyre, C.C., and Salmon, H.P.
1999. Differential recruitment of T- and IgA B-lymphocytes in the developing mammary gland in relation to homing receptors and vascular addressins. J. Histochem. Cytochem. 47:1581-1592.

34. Yang, X.D., et al. 1994. A predominant role of integrin alpha 4 in the spontaneous development of autoimmune diabetes in nonobese diabetic mice. Proc. Natl. Acad. Sci. U. S. A. 91:12604-12608.

35. Rott, L.S., Briskin, M.J., Andrew, D.P., Berg, E.L., and Butcher, E.C. 1996 A fundamental subdivision of circulating lymphocytes defined by adhesion to mucosal addressin cell adhesion molecule-1. Comparison with vascular cell adhesion molecule- 1 and correlation with beta 7 integrins and memory differentiation. J. Immunol. 156:3727-3736.

36. Bentley, A.M., et al. 1993. Expression of endothelial and leukocyte adhesion molecules interacellular adhesion molecule-1, E-selectin, and vascular cell adhesion molecule- 1 in the bronchial mucosa in steady-state and allergen-induced asthma. J. Allergy Clin. Immunol. 92:857-868.

37. Stavnezer, J. 1995. Regulation of antibody production and class switching by TGF-beta. J. Immunol. 155:1647-1651.

38. Calame, K.L. 2001. Plasma cells: finding new light at the end of B cell development. Nat. Immunol. 2:1103-1108.

39. Homey, B., et al. 2002. CCL27-CCR10 interactions regulate T cell-mediated skin inflammation. Nat. Med. 8:157-165.

40. Power, C.A., et al. 1997. Cloning and characterization of a specific receptor for the novel CC chemokine MIP-3alpha from lung dendritic cells. J. Exp. Med. 186:825-835.

41. Liao, F., et al. 1999. CC-chemokine receptor 6 is expressed on diverse memory subsets of $\mathrm{T}$ cells and determines responsiveness to macrophage inflammatory protein 3 alpha. J. Immunol. 162:186-194.

42. McHeyzer-Williams, M.G., and Ahmed, R. 1999. B cell memory and the long-lived plasma cell. Curr. Opin. Immunol. 11:172-179.

43. Hargreaves, D.C., et al. 2001. A coordinated change in chemokine responsiveness guides plasma cell movements. J. Exp. Med. 194: $45-56$ 